\title{
Research on the Precession Characteristics of Hemispherical Resonator Gyro
}

\author{
Li-Jun Song $\mathbb{D}^{1},{ }^{1}$ Rui Yang, ${ }^{1}$ Wang-Liang Zhao, ${ }^{2}$ Xing He$^{1}{ }^{\text {Shaoliang Li, }}{ }^{2}$ and You-Jun Ding ${ }^{1}$ \\ ${ }^{1} X i$ 'an University of Architecture and Technology, School of Information \& Control Engineering, Xi'an, Shaanxi 710055, China \\ ${ }^{2}$ Shanghai Aerospace Control Technology Institute, Shanghai 201109, China \\ Correspondence should be addressed to Li-Jun Song; songlijun9071@sina.com
}

Received 18 September 2020; Revised 16 October 2020; Accepted 27 November 2020; Published 28 January 2021

Academic Editor: Ning Wang

Copyright (c) 2021 Li-Jun Song et al. This is an open access article distributed under the Creative Commons Attribution License, which permits unrestricted use, distribution, and reproduction in any medium, provided the original work is properly cited.

Hemispherical Resonator Gyro (HRG) is a new type gyro with high precision, high reliability, shock resistance, no need of preheating, short start time, and long life. It is a kind of vibrating gyro with standing wave rotating along the sensitive base of annular precession, has a unique application prospect in the field of high precision inertial sensors, and is widely used in unmanned aerial vehicle control in complex environments. Based on the theory of the structure characteristics of the hemispherical resonator, the mathematical model of energy of the resonator is established to research the rule of resonant frequency when the hemispherical resonator is rotated around the central axis. In this paper, the influence of precession factor, which are the top angle, the bottom angle, and wall unevenness of the hemispherical resonator, are analyzed. A series of hemispherical resonator models are constructed by ANSYS software to prove the results of theoretical research. The simulation results show that precession factor of the hemispherical resonator is more sensitive of the top angle than the bottom angle, and the error of angular velocity which is caused by the change of the top angle is larger than that which is caused by the change of the bottom angle.

\section{Introduction}

The main sensitive part of the HRG is the hemispherical resonator with ultralow damping [1-3]. It is the superior performance of the hemispherical resonator that the HRG which can be widely used in the fields of land, sea, air, and sky electricity. In this paper, the characteristics of the HRG are analyzed with the resonator forming process, performance indicators, and user requirements, the mathematical model of the resonator is established, and the optimum mode and precession factor are determined. It provides the theoretical research basis for large quantity and low cost, so as to accelerate the development and application process of the HRG.

\section{The Equation of Motion of Resonator}

The hemispherical resonator consists of two parts: the thinwalled hemispherical shell and the supporting rod. The resonator material reaches the maximum value of bending energy storage because of the thin-walled hemispherical shell, and the support rod is fixed by indium welding, which acts as a constraint and supports the resonator and also carries on the transmission of electrical signals. The paper discussed the mathematical model of the energy of the hemispherical resonator based on the Kirchhoff and gave the specific expressions of resonant frequency and precession factor based on the principle of energy conservation. The influence of resonator parameters on the precession factor caused by process defects such as the bottom angle $\varphi_{0}$, top angle $\varphi_{F}$, and nonideal wall thickness $h(\varphi)$ is also analyzed by using the mathematical model of the energy resonator.

The coordinates of the hemispherical resonator is shown in Figure $1[4,5]$.

The $x$-axis is the central axis of the resonator; $R$ and $h(\varphi)$ are the center radius and wall thickness of the hemispherical shell, respectively, $\varphi_{0}$ is the bottom angle of the resonator, which is the angle between the constrained end of the resonator and the center axis, which is determined by the ratio of the radius of the supporting rod of the resonator to 


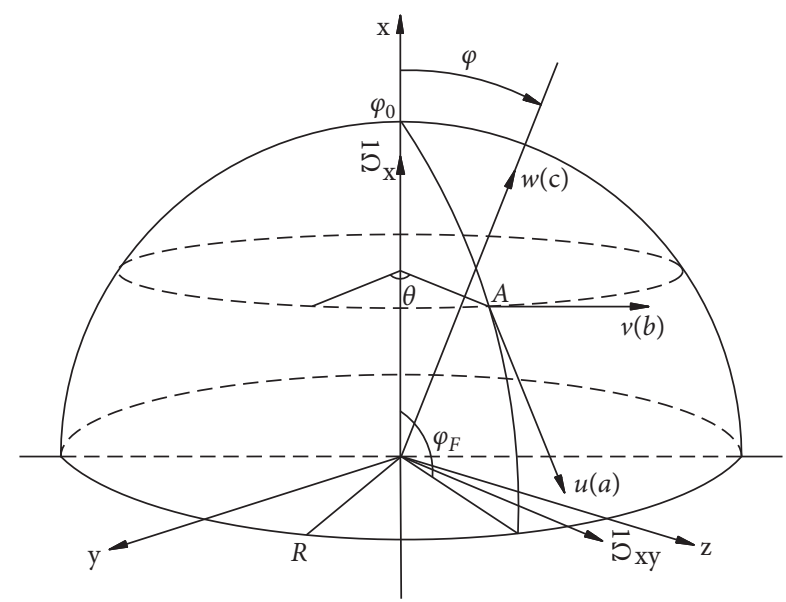

FIgURE 1: The coordinate of the hemispherical resonator.

the radius of the hemispherical shell, and $\varphi_{F}$ is the top angle of the resonator, which is the angle between the free end of the resonator and the center axis without any constraint, $\varphi_{0}=0^{\circ}$ and $\varphi_{F}=90^{\circ}$.

The displacement vector of point $\mathrm{A}$ on the shell is

$$
\mathbf{V}=\mathbf{a} u+\mathbf{b} v+\mathbf{c} w
$$

where $u, v$, and $w$ are the displacement components of shell, tangent, and radial, respectively, and $\mathbf{a}, \mathbf{b}$, and $\mathbf{c}$ are the corresponding dynamic vectors, respectively.

The deformation and stress of the resonator are constrained by the elastic mechanics of the thin shell. Assuming that the resonator rotates at an angular velocity $\Omega=\Omega_{x}+$ $\Omega_{y z}$ in the inertial space, the mode of the resonator in the rotational space can be expressed as follows:

$$
\begin{aligned}
& \left\{\begin{array}{l}
u(\varphi, \theta, t)=u(\varphi) \cos n(\theta+\psi) \cos \omega_{n} t \\
v(\varphi, \theta, t)=v(\varphi) \sin n(\theta+\psi) \cos \omega_{n} t \\
w(\varphi, \theta, t)=w(\varphi) \cos n(\theta+\psi) \cos \omega_{n} t
\end{array}\right. \\
& \psi=\int_{t_{0}}^{t} \mathrm{Pd} t=K \int_{t_{0}}^{t} \Omega \mathrm{d} t
\end{aligned}
$$

where $u(\varphi), v(\varphi)$, and $w(\varphi)$ are the modes distributed along each direction; $\psi$ is the precession angle of the modes; $n$ is circumferential waves; $\omega_{n}$ is the resonant frequency; $K$ is the precession factor.

According to the theory of elastic thin shell, the elastic potential energy of the hemispherical resonator is as follows:

$$
U=\frac{E}{2\left(1-\mu^{2}\right)} \int_{S}\left[\varepsilon_{\theta}^{2}+\varepsilon_{\varphi}^{2}+2 \mu \varepsilon_{\theta} \varepsilon_{\varphi}+\frac{1-\mu}{2} \varepsilon_{\varphi \theta}^{2}+\frac{h^{2}(\varphi)}{12}\left(\lambda_{\theta}^{2}+\lambda_{\varphi}^{2}+2 \mu \lambda_{\theta} \lambda_{\varphi}+\frac{1-\mu}{2} \lambda_{\varphi \theta}^{2}\right)\right] h(\varphi) \mathrm{d} S,
$$

where $E$ is the elastic modulus and $\mu$ is Poisson's ratio of the material.
In the hemispherical shell, the midplane strain and the midplane bending deformation of the stretchable hemispherical thin shell in formula (3) are as follows:

$$
\begin{aligned}
& \left\{\begin{array}{l}
\varepsilon_{\varphi}=\frac{(\partial u / \partial \varphi+w)}{R}, \\
\varepsilon_{\theta}=\frac{(\partial v / \partial \theta+u \cos \varphi+w \sin \varphi)}{R \sin \varphi}, \\
\varepsilon_{\varphi \theta}=\frac{(\partial u / \partial \theta+\partial v / \partial \varphi \sin \varphi-v \cos \varphi)}{R \sin \varphi}, \\
\lambda_{\varphi}=\frac{\left(\partial u / \partial \varphi-\partial^{2} w / \partial \varphi^{2}\right)}{R^{2}}, \\
\lambda_{\varphi \theta}=\frac{\left[\partial u / \partial \theta+\sin \varphi \partial v / \partial \varphi-v \cos \varphi+2\left(\cos \varphi / \sin \varphi \partial w / \partial \theta-\partial^{2} w / \partial \varphi \partial \theta\right)\right]}{\left(R^{2} \sin \varphi\right)} .
\end{array}\right. \\
& \left.R^{2} \varphi \partial^{2} w / \partial \theta^{2}-\cos \varphi \partial w / \partial \varphi+u \cos \varphi+\partial v / \partial \theta\right) \\
&
\end{aligned}
$$


In the practical system, the top of the hemispherical shell is free and the bottom is constrained and is in the state of microamplitude vibration, so the hemispherical shell satisfies the theory that the normal and tangential strain of the middle plane is zero. $u(\varphi), v(\varphi)$, and $w(\varphi)$ have the following relations:

$$
\left\{\begin{array}{l}
w(\varphi)=-\frac{d u(\varphi)}{d \varphi} \\
n v(\varphi)+u(\varphi) \cos (\varphi)-\frac{d u(\varphi)}{d \varphi} \sin \varphi=0 \\
n u(\varphi)+v(\varphi) \cos (\varphi)-\frac{d v(\varphi)}{d \varphi} \sin \varphi=0
\end{array}\right.
$$

Due to the constraint boundary condition at the bottom,

$$
u\left(\varphi_{0}\right)=v\left(\varphi_{0}\right)=w\left(\varphi_{0}\right)=0
$$

it can be obtained

$$
\left\{\begin{array}{l}
u(\varphi)=v(\varphi)=C_{1} \sin \varphi \tan ^{n} \frac{\varphi}{2} \\
w(\varphi)=-C_{1}(n+\cos \varphi) \tan ^{n} \frac{\varphi}{2}
\end{array}\right.
$$

where $C_{1}$ is a constant to be determined by the initial excitation condition of the vibration [6-9].

\section{The Precession Characteristics of the HRG}

3.1. The Working Principle of HRG. HRG is measured by the angular velocity or angle of the input based on the Coriolis principle. When HRG does not rotate, the position of the wave belly point and the wave node is stationary relative to the hemispherical shell, and when the external excitation is applied to the resonator, the energy of the resonator can be converted between different modes to form a stable vibration shape. When the HRG is rotated, the resonant shape of the stable vibration will lag the physical rotation of the gyroscope body, and its hysteresis is about 0.3 times of the rotation angle; the principle is shown in Figure 2.
3.2. The Resonant Frequency of the HRG. When the resonant frequency of the hemispherical resonator is researched, the inertial force acting on the hemispherical resonator should be considered, and it is also necessary to consider the external force acting on the edge of the surface of the resonator.

Assuming the elastic force of the virtual work is $\delta W_{K}$, external force caused by angular velocity $\Omega$ of the virtual work is $\delta W_{e}$ and vibration inertia force of the virtual work is $\delta T$.

According to the reference [10], with the displacement $V$, the virtual work of the vibration inertia force $F$ can be obtained as follows:

$$
\begin{aligned}
\delta T= & \int V d F \\
= & \rho \pi R^{2} e^{2 i \omega_{n} t} \int_{\varphi_{0}}^{\varphi_{F}}\left(n^{2} P^{2}+\omega^{2}\right)\left(\sin ^{2} \varphi+2 n \cos \varphi\right. \\
& \left.+n^{2}+1\right) \tan ^{2 n}\left(\frac{\varphi}{2}\right) \sin \varphi h(\varphi) \mathrm{d} \varphi C_{1} \delta C_{1} .
\end{aligned}
$$

The external force caused by the angular velocity needs to be considered because the rotating inertia force causes the rotating acceleration $\mathbf{a}(\Omega)=\mathbf{a}\left(\Omega_{x}\right)+\mathbf{a}\left(\Omega_{y z}\right)$. The virtual work of the rotating inertia force can be obtained as follows:

$$
\begin{aligned}
\delta W_{e}= & \rho \pi R^{2} e^{2 i \omega_{n} t} \int_{\varphi_{0}}^{\varphi_{F}}\left[-4 n^{2} \sin ^{3} \varphi \tan ^{2 n}\left(\frac{\varphi}{2}\right) \Omega_{x} P\right. \\
& \left.+\sin ^{3} \varphi\left(1+n^{2}\right) a n^{2 n}\left(\frac{\varphi}{2}\right) \Omega_{x}^{2}+f_{1}(n, \varphi) \Omega_{y z}^{2}\right] \\
& \cdot h(\varphi) \mathrm{d} \varphi C_{1} \delta C_{1}, \\
f_{1}(n, \varphi)= & \frac{1}{2}\left(\sin ^{2} \varphi\left(3+2 n \cos \varphi+\cos ^{2} \varphi\right)\right. \\
& \left.+\left(\cos ^{2} \varphi+n\right)^{2}\left(1+\cos ^{2} \varphi\right)\right) \tan ^{2 n}\left(\frac{\varphi}{2}\right) .
\end{aligned}
$$

The elastic potential energy of the shell is produced by the elastic force, and the virtual work of the elastic force is as follows:

$$
\delta W_{K}=\delta(-U)
$$

According to the reference [10], the virtual work of elastic force can be obtained as follows:

$$
\begin{aligned}
\delta W_{K} & =-\pi e^{2 i \omega_{n} t}\left\{\int_{\varphi_{0}}^{\varphi_{F}} \frac{E}{(1+\mu) R^{2}} \frac{n^{2}\left(n^{2}-1\right)^{2}}{3 \sin ^{3} \varphi} \tan ^{2 n}\left(\frac{\varphi}{2}\right) h^{3}(\varphi) \mathrm{d} \varphi+R^{2} \rho \int_{\varphi_{0}}^{\varphi_{F}}\left[\left(f_{2}(n, \varphi) \Omega_{x}^{2}+f_{3}(n, \varphi) \Omega_{y z}^{2}\right)\right] h(\varphi) \mathrm{d} \varphi\right\} C_{1} \delta C_{1}, \\
f_{2}(n, \varphi) & =(n+\cos \varphi)^{2}\left(\sin ^{2} \varphi+n^{2}\right)\left(\varphi \cos \varphi+\sin ^{2} \varphi\right) \tan ^{2 n}\left(\frac{\varphi}{2}\right)+\sin ^{4} \varphi\left(\sin ^{2} \varphi-2 n(n+\cos \varphi)\right) \tan ^{2 n}\left(\frac{\varphi}{2}\right), \\
f_{3}(n, \varphi) & =\frac{1}{2}\left(n^{2}+\sin ^{2} \varphi\right)(\cos \varphi+n)^{2}\left(\varphi \cos \varphi+1+\cos ^{2} \varphi\right) \tan ^{2 n}\left(\frac{\varphi}{2}\right)+\left(\sin ^{2} \varphi\left(\cos ^{2} \varphi+1\right)\right)\left(\sin ^{2} \varphi-2 n(n+\cos \varphi)\right) \tan ^{2 n}\left(\frac{\varphi}{2}\right) .
\end{aligned}
$$




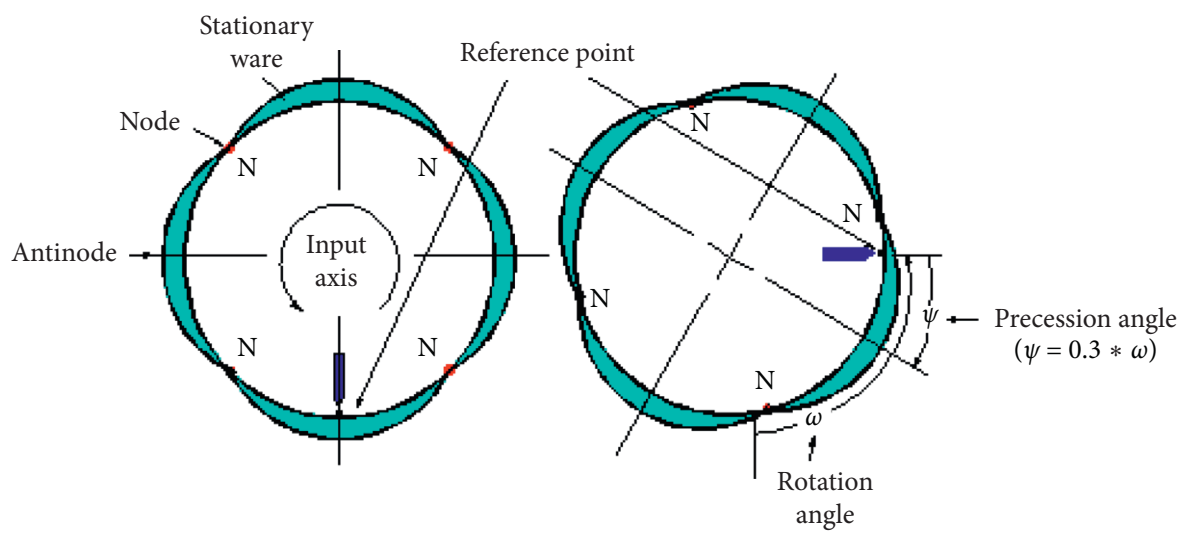

FIgURE 2: The angle hysteresis principle of the hemispherical resonator.

According to the principle of virtual displacement, the total sum of work by virtual displacement is zero, it remains still is that and the result can be obtained:

$$
\delta T+\delta W_{e}+\delta W_{K}=0
$$

The frequency of the hemispherical resonator is as follows:

$$
\omega_{n}=\sqrt{\frac{k_{0}}{k_{m}}},
$$

where

$$
\begin{aligned}
& k_{m}=\int_{\varphi_{0}}^{\varphi_{F}}\left(\sin ^{2} \varphi+2 n \cos \varphi+n^{2}+1\right) \tan ^{2 n}\left(\frac{\varphi}{2}\right) \sin \varphi h(\varphi) \mathrm{d} \varphi, \\
& k_{0}=\frac{E n^{2}\left(n^{2}-1\right)^{2}}{\rho(1+\mu) R^{4}} \int_{\varphi_{0}}^{\varphi_{F}} \frac{1}{3 \sin ^{3} \varphi} \tan ^{2 n}\left(\frac{\varphi}{2}\right) h(\varphi) \mathrm{d} \varphi .
\end{aligned}
$$

3.3. The Precession Factor of the HRG Mode. When the HRG rotates at an angular velocity $\Omega$ in the inertial space, the circular mode turns the $\psi$ angle in reverse at the rate $P=$ $K \Omega_{x}[11,12]$.

The inertial forces at any point in the hemispherical resonator shell are

$$
F=F_{0}+F\left(\Omega_{x}\right)+F\left(\Omega_{y z}\right),
$$

where $F_{0}, F\left(\Omega_{x}\right)$, and $F\left(\Omega_{y z}\right)$ are the inertia forces when the hemispherical resonator does not rotate, respectively, and the virtual work of the virtual displacement is

$$
\delta T=\delta T_{0}+\delta T\left(\Omega_{x}\right)+\delta T\left(\Omega_{y z}\right)+\delta T(\Omega)-\delta W(\Omega),
$$

where

$$
\begin{aligned}
\delta T_{0}= & \omega^{2} \pi \rho \cos ^{2} \omega t \int_{\varphi_{0}}^{\varphi_{F}}[u(\varphi) \delta u+v(\varphi) \delta v+w(\varphi) \delta w] R h(\varphi) \mathrm{d} \varphi, \\
\delta T\left(\Omega_{x}\right)= & n^{2} P^{2} \pi \rho \cos ^{2} \omega t \int_{\varphi_{0}}^{\varphi_{F}}[u(\varphi) \delta u+v(\varphi) \delta v+w(\varphi) \delta w] R h(\varphi) \mathrm{d} \varphi+2 \pi \rho n P \Omega_{x} \cos ^{2} \omega t \\
& \cdot \int_{\varphi_{0}}^{\varphi_{F}}[\cos \varphi v(\varphi) \delta u+\cos \varphi u(\varphi) \delta v+\sin \varphi v(\varphi) \delta w+\sin \varphi w(\varphi) \delta v] R h(\varphi) \mathrm{d} \varphi, \\
\delta T\left(\Omega_{y z}\right)= & 0 .
\end{aligned}
$$

$\delta W(\Omega)$ is the virtual work of the initial elastic force caused by the $\Omega$, that is, $\delta W_{K 0}$, and it can be obtained from the principle of virtual displacement:

$$
\delta T+\delta W_{K 0}=0
$$

that is,

$$
\delta W_{K 0}-\delta W_{K}(\Omega)+\delta T_{0}+\delta T(\Omega)=0
$$

In the vibration mode of the resonator, $\delta W_{K 0}-\delta W_{K}(\Omega)$ is the virtual work of modal elastic force and $\delta T_{0}$ is the virtual work of elastic force. When the HRG rotates at angular velocity $\Omega=\Omega_{x}+\Omega_{y z}$ in the inertial space, $\Omega_{x}$ is the main vibration. According to the principle that the main vibration energy remains unchanged, when the hemispherical resonator shell rotates, the sum of the main vibration energy and potential energy remains unchanged, and it can be obtained 


$$
\delta T\left(\Omega_{x}\right)=0
$$

Suppose

$$
\begin{aligned}
G_{1}= & \int_{\varphi_{0}}^{\varphi_{F}}[\cos \varphi v(\varphi) \delta u+\cos \varphi u(\varphi) \delta v+\sin \varphi v(\varphi) \delta w \\
& +\sin \varphi w(\varphi) \delta v] \operatorname{Rh}(\varphi) \mathrm{d} \varphi, \\
G_{2}= & \int_{\varphi_{0}}^{\varphi_{F}}[u(\varphi) \delta u+v(\varphi) \delta v+w(\varphi) \delta w] R^{2} h(\varphi) \mathrm{d} \varphi .
\end{aligned}
$$

Then,

$$
n P G_{1}+2 G_{2} \Omega_{x}=0 .
$$

The precession factors can be obtained by finishing:

$$
K=\frac{P}{\Omega_{x}}=-\frac{2}{n} \frac{G_{2}}{G_{1}},
$$

where $G_{1}$ and $G_{2}$ are only related to the shape of vibration, the constraint relation of resonator, and the geometric properties of shell; it also reflects the Coriolis effect and inertial force on the shell.

\section{The Parameter of Resonator Effect on the Parameter of the HRG}

According to the requirement of high precision, assuming the radius is $15 \mathrm{~mm}$, the error of spherical shell radius is not bigger than $0.014 \%$ (about $2 \mu \mathrm{m}$ ) to ensure the stability of the resonant frequency. The thickness of resonator wall is $0.6 \mathrm{~mm}$, and the error is not bigger than $0.067 \%$, about $0.402 \mu \mathrm{m}$. Spherical shell sphericity is not bigger than $0.0003 \mathrm{~mm}$. Bracket radius is $2 \mathrm{~mm}$, and the error support rod is not bigger than $0.13 \%$, about $7.8 \mu \mathrm{m}$. The bottom angle $\varphi_{0}=7.3^{\circ}$ can be obtained from the ratio of the radius of the supporting rod and the hemispherical shell. The precision of the top angle and the bottom angle is not greater than $0.01^{\circ}$; the brace bar and hemispherical coaxiality are not bigger than $0.0015 \mathrm{~mm}$, and the quality factor $Q$ is not less than $1 \times 10^{7}$.

4.1. The Optimal Number of Circumferential Waves. Equations (13) and (23) were used to calculate the resonant frequency and precession factor of the hemispherical resonator, as shown in Table 1.

With the increase of the number of circumferential wave number $n$, the mode of the resonator is becoming more and more complex, and the resonant frequency and the energy which is to maintain the resonator vibration is also increasing continuously. The precession factor of the hemispherical resonator decreases with the number of circumferential wave.

From the view of the energy loss of the hemispherical resonator, it is hoped that less energy is needed to maintain the mode of hemispherical resonator. Considering the ap-

\begin{tabular}{|c|c|c|c|c|}
\hline & $n=2$ & $n=3$ & $n=4$ & $n=5$ \\
\hline$\omega_{n}\left(\mathrm{H}_{\mathrm{Z}}\right)$ & 9 & 2804 & 5620 & 9307 \\
\hline$K \begin{array}{c}\varphi_{0}=0^{\circ} \\
\varphi_{0}=7.3^{\circ}\end{array}$ & $\begin{array}{l}-0.2978239 \\
-0.2981762\end{array}$ & $\begin{array}{l}-0.0659867 \\
-0.0659901\end{array}$ & $\begin{array}{l}-0.0231877 \\
-0.0231877\end{array}$ & $\begin{array}{l}-0.0102203 \\
-0.0102203\end{array}$ \\
\hline
\end{tabular}
plication and vibration realization condition of the HRG, the optimal number of circumferential waves is $n=2$, and at this time, $K \approx 0.3$.
TABLE 1: The frequency and precession factor of the hemispherical resonator

4.2. Effect of Top Angle and Bottom Angle on Precession Factor. Assuming the change rate of the mode precession factor $\mathrm{K}$ of the resonator is

$$
\sigma_{K}=\frac{\Delta K}{K_{0}}
$$

where $K_{0}$ is the precession factor when the structural parameters of the resonator meets the requirement of design $\left(\varphi_{0}=7.3^{\circ}\right.$ and $\left.\varphi_{F}=90^{\circ}\right)$ and $\Delta K$ is the variable of precession factor $[13,14]$.

The precession factor $K$ is

$$
K=-\frac{2}{n} \frac{\int_{\varphi_{0}}^{\varphi_{F}} 4 \sin ^{2} \varphi \cos \varphi \tan ^{n} \varphi / 2 h(\varphi) \mathrm{d} \varphi}{\int_{\varphi_{0}}^{\varphi_{F}}\left(n^{2}+2 n \cos \varphi+\cos ^{2} \varphi-3 \sin ^{2} \varphi\right) \tan ^{n} \varphi / 2 h(\varphi) \mathrm{d} \varphi} .
$$

When the number of circumferential waves is 2 , the relationship between $\varphi_{F}$ and $\varphi_{0}$ of the resonator and $\mathrm{K}$ is shown in Figures 3 and 4:

When the structure parameters of the resonator meet the requirement of design, that is, $\varphi_{0}=7.3^{\circ}$ and $\varphi_{F}=90^{\circ}$, the precession factor is $K_{0}=-0.2981762$; when the change is $\left[7^{\circ}, 8^{\circ}\right], \sigma_{K}$ change is $[-0.0139 \%, 0.00037 \%]$, and the precession factor $K$ is insensitive to the change of the bottom angle; while when the change is $\left[89^{\circ}, 91^{\circ}\right]$, change of $\sigma_{K}$ is $[1.2425 \%,-1.4761 \%]$, and the precession factor $K$ is relatively sensitive to the change of the top angle.

The design requires that the precision of the top angle is not bigger than 0.01 .

When $\Delta \varphi_{0}=0.01^{\circ}$ and $\Delta K=1.2 \times 10^{-6}$, if the input angular velocity is $1^{\circ} / \mathrm{s}$, the error of angular velocity caused by $\Delta K$ is $\Delta \Omega=0.00432^{\circ} / \mathrm{h}$.

When $\Delta \varphi_{F}=0.01^{\circ}$ and $\Delta K=4 \times 10^{-5}$, if the input angular velocity is $1^{\circ} / \mathrm{s}$, the error of angular velocity caused by $\Delta K$ is $\Delta \Omega=0.144^{\circ} / h$.

It can be seen that the error of angular velocity caused by $\Delta \varphi_{0}$ is far less than that caused by the same $\Delta \varphi_{F}$.

4.3. Effect of Uneven Wall Thickness on Precession Factor. The processing of high-precision spherical shell structure is the core of the hemispherical resonator gyro technology. According to the material and structural characteristics of the hemispherical resonator, the structure of the resonator is analyzed, which is combined with the technical bottleneck existing in the processing of fused quartz glass, and the processing performance is studied.

For the vibration mode with fixed number of circumferential waves, the uneven wall thickness caused by machining will cause the change of resonance frequency and also affects the precession factor. The influence can be 


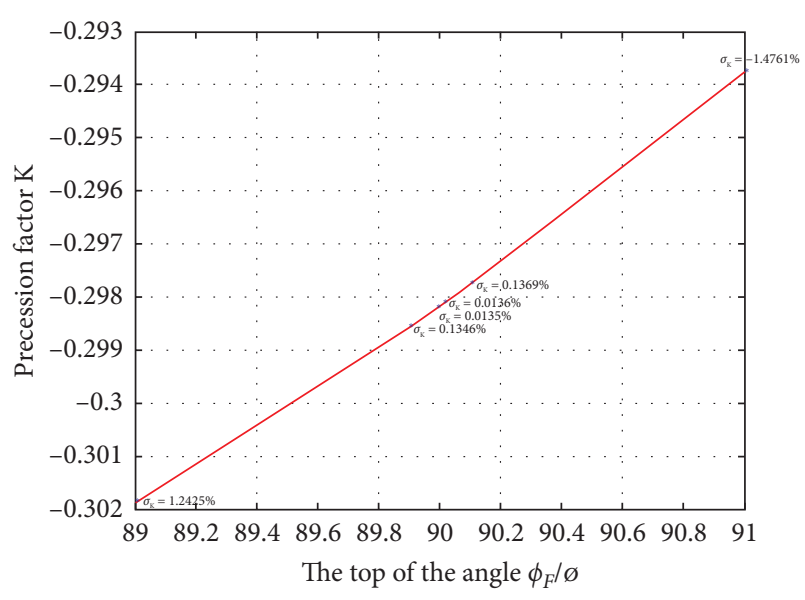

Figure 3: The relation between $\varphi_{F}$ and $K\left(\varphi_{0}=7.3^{\circ}\right)$.

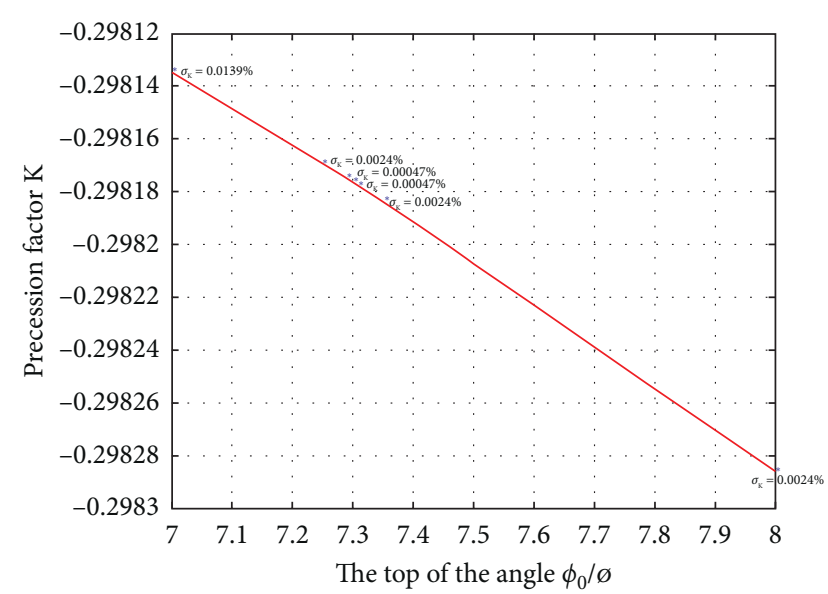

Figure 4: The relation between $\varphi_{0}$ and $K\left(\varphi_{F}=90^{\circ}\right)$.

divided into two cases. (1) When the wall thickness of the bottom of the resonator increases, the variation of the wall thickness is

$$
h(\varphi)=h(1+\alpha \cos \varphi)
$$

(2) When the wall thickness of the top of the resonator increases, the function of the wall thickness is

$$
h(\varphi)=h(1+\alpha \sin \varphi) .
$$

The change of spherical angle $\varphi$ of the resonator is $0^{\circ} \leq \varphi \leq 90^{\circ}$, and $\alpha$ is the change factor of wall thickness.

When the mode shape circumferential wave number is 2 , the wall thickness of the bottom and top increases by $K$; the changes are shown in Figures 5 and 6.

The absolute value of the precession factor $K$ decreases with the increase of the wall thickness of the bottom. When the change of $\alpha$ is $[0,0.25]$, the change of $\sigma_{K}$ is $[-0.012308 \%,-1.1021 \%]$.

The absolute value of the precession factor $K$ increases with the increase of the wall thickness of the bottom. When the change of $\alpha$ is $[0,0.25]$, the change of $\sigma_{K}$ is [0.0126435\%, 1.0503186\%].

\section{Design and Analysis of Simulation}

5.1. Finite Element Analysis (FEA) of the Resonator. FEA is used to simulate and analyze the actual physical system with the mathematical approximation method. The characteristic changes caused by the rotation of the hemispherical resonator will affect the performance of the HRG, especially the structure of the hemispherical resonator is the key factor affecting the vibration characteristics of the HRG.

Assuming the hemispherical resonator is damped or damped in a free state without rotation, the differential equation of multi-degree-of-freedom motion is

$$
M \ddot{x}(t)+C \dot{x}(t)+K x(t)=Q(t),
$$

where $\ddot{x}(t)$ is the acceleration vector, $\dot{x}(t)$ is the velocity vector, $x(t)$ is the displacement vector, and $Q(t)$ is the resonator node load vector. $\mathrm{M}, \mathrm{C}$, and $\mathrm{K}$ are the mass matrix, damping matrix, and stiffness matrix of the hemispherical resonator, respectively [15].

Equation (29) in the time domain is transformed into a complex variable in the Laplace domain $p$, and the initial displacement and velocity are assumed to be zero, then the Laplace domain equation is obtained:

$$
M p^{2}+C p+K=Q(p) .
$$

The transfer function is

$$
H(p)=\frac{1 / M}{p^{2}+(K / M) p+(K / M)} .
$$

Its root is the pole:

$$
\lambda_{1,2}=-\left(\frac{C}{(2 M)}\right) \pm \sqrt{\left(\frac{C}{(2 M)}\right)^{2}-\left(\frac{K}{M}\right)}
$$

The natural frequencies are defined as $\omega_{n}=\sqrt{K / M}$.

It is assumed that the energy loss of the hemispherical resonator is very small during an oscillation period, and the damping of the hemispherical resonator is approximately ignored in the analysis. The differential equation is simplified as follows:

$$
M \ddot{x}(t)+K x(t)=Q(t) .
$$

When the node load vector of the hemispherical resonator is 0 , the differential equation is further simplified as follows:

$$
M \ddot{x}(t)+K x(t)=0 .
$$

Equation (33) is the free vibration equation of the hemispherical resonator, also known as the dynamic characteristic equation. The natural frequencies and modes of the resonator can be solved by this equation. 


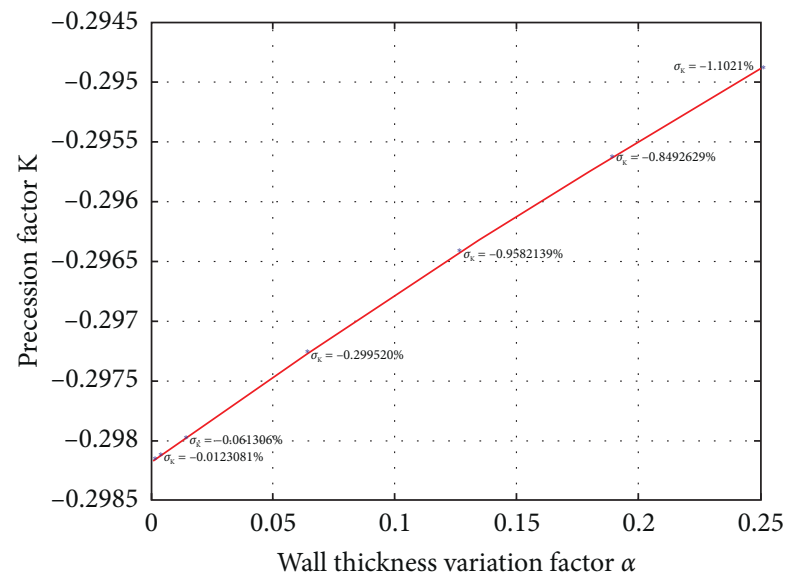

FIgURE 5: The precession factor with increased thickness of the bottom.

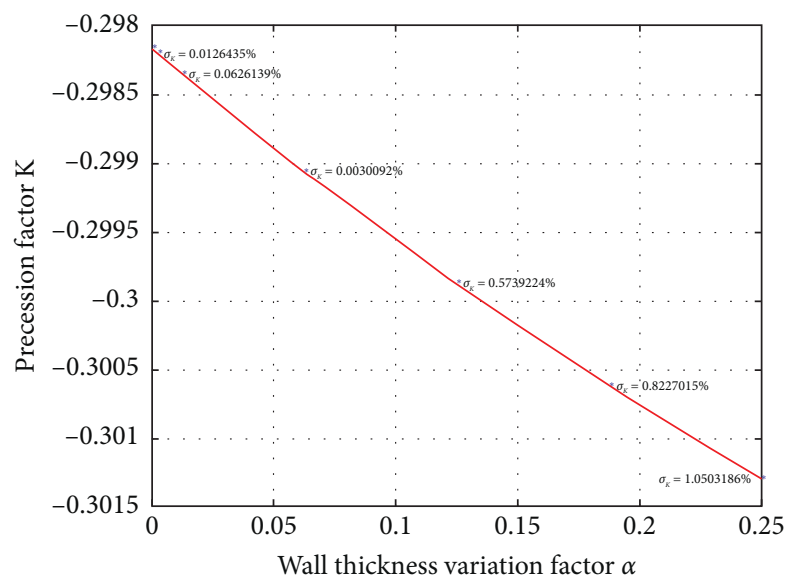

Figure 6: The precession factor with increased thickness of the top.

5.2. Finite Element Simulation of Resonator. For high-quality hemispherical resonator, on the one hand, it is necessary to ensure the stability of resonant frequency and, on the other hand, to ensure the stability of mode. At present, the ideal material of the hemispherical resonator is fused silica glass. The hemispherical resonator made of this material has stable vibration frequency, short delay time, high mechanical stability, low thermal expansion rate, and large internal stress. The hemispherical resonator made of fused silica glass can work in a wide temperature range and reduces the energy loss needed to maintain the gyro vibration.

The hemispherical resonator requires the fused silica glass to have isotropic and high quality factor, and the temperature coefficient is small and stable; usually, elastic modulus is $E=7.67 \times 10^{10} \mathrm{~Pa}$, Poisson's ratio is $\mu=0.17$, and density is $\rho=2500 \mathrm{~kg} / \mathrm{m}^{3}$. In this paper, the boundary constraint of the hemispherical resonator gyro is that the bottom is fixed and the top is free. The specific method used in the simulation is to constrain all the degrees of freedom of the upper end of the support in ANSYS, and the resonator and the following components adopt the free state.

To reduce the workload of the hemispherical resonator modeling, AutoCAD is used to establish the model and then imported into the ANSYS. Finally, the grid generation, hemispherical resonator model, and grid generation are mapped, as shown in Figures 7(a) and 7(b).

When the bottom of the middle support of the hemispherical resonator is fixed, a force is applied to the hemispherical resonator. The results show that the color of the resonator changes with the color of the lower part of the strut. From the total deformation level of the resonator, the deformation of the hemispherical resonator and the rod is closer to the edge of the hemisphere, and the greater the deformation, the greater the tendency of gradual increase. The total deformed flat view and bottom view of the hemispherical resonator are shown in Figures 8(a) and 8(b).

Under the condition that the bottom of the middle supporting rod of the hemispherical resonator is fixed and the top is free, the stress change is the largest between the hemispherical resonator and the rod, and it gradually decreases from the supporting rod to the outside. The contact surface of the central rod and the hemispherical resonator is the place where the stress changes greatly, and it extends from inside to outside, and with the movement of the hemispherical resonator, some deformation occurs. The resonator's potential view and bottom view are shown in Figures 9(a) and 9(b). 


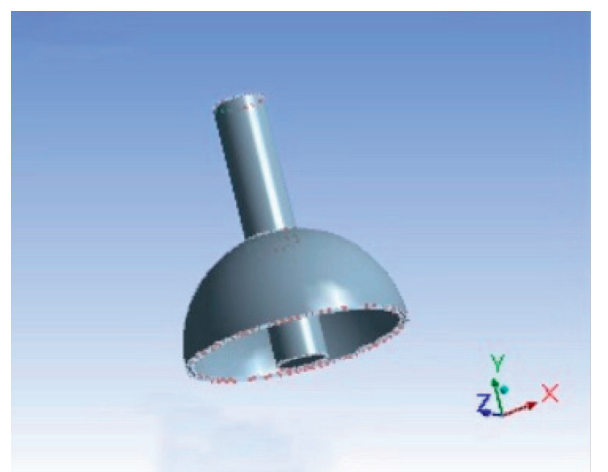

(a)

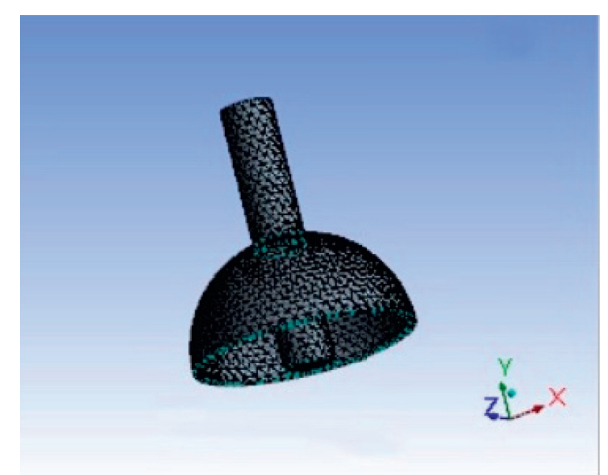

(b)

FIgURE 7: The grid and model of the hemispherical resonator.

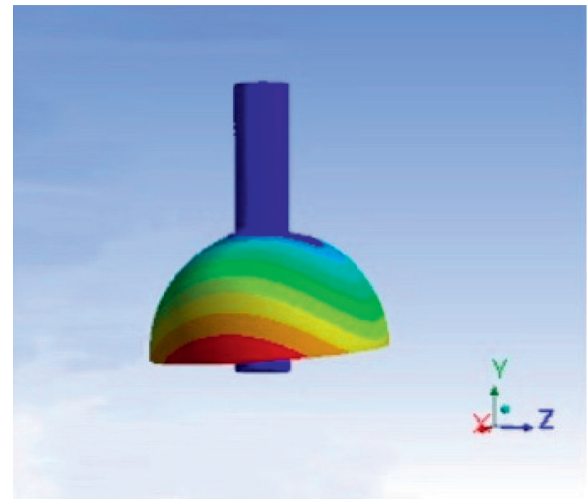

(a)

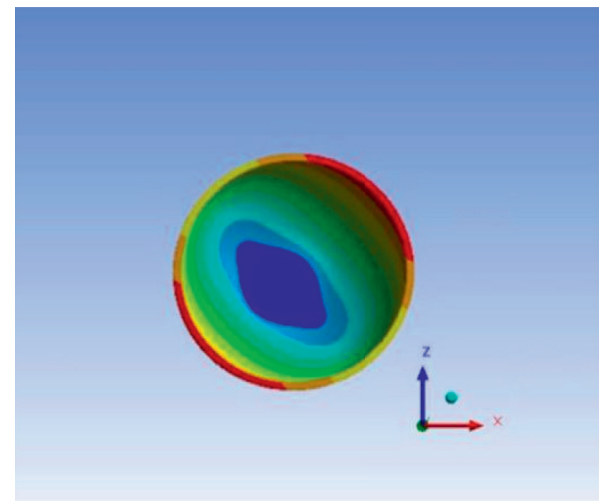

(b)

FIgURE 8: The plane view and bottom view of the hemispherical resonator's deformation.

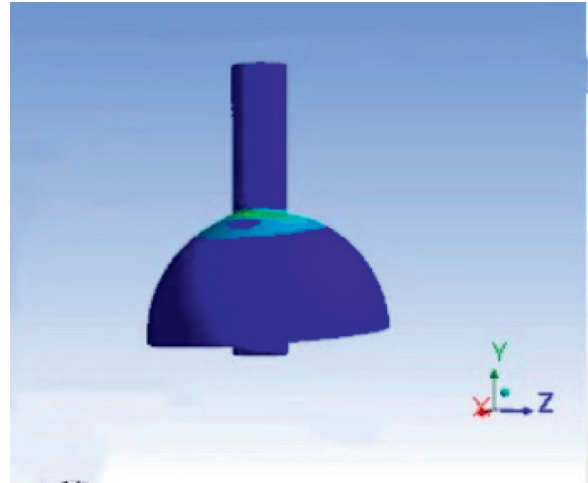

(a)

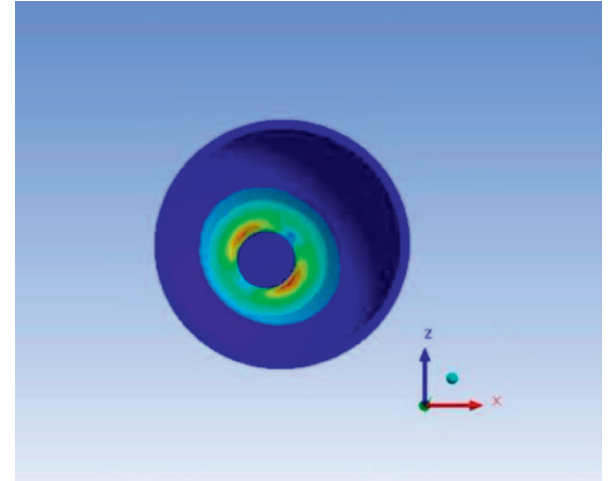

(b)

FIgURE 9: The plane view and bottom view of hemispherical resonator's SEQV.

\section{Conclusion}

The mathematical model of the energy resonator is presented in the paper. The influence of nonideal factors on resonant frequency and precession factor is studied, and the influence of nonideal factors on the precession factor is analyzed. The precession factor of the HRG is more sensitive to the change of the top angle than to the change of the bottom angle. The angular velocity error of the HRG caused by the change of the top angle is much larger than that caused by the change of the bottom angle. Therefore, in the actual manufacturing process, the manufacturing precision of the top corner is much higher than that of the bottom corner. With the increase of the thickness of the 
hemispherical resonator, the stiffness and resonant frequency of the hemispherical resonator will increase. The increase of the resonant frequency makes the hemispherical resonator difficult to excite. However, when the wall thickness of the hemispherical shell decreases, the measurement error will increase due to the asymmetry. Therefore, in the actual manufacturing process, the selection of wall thickness needs comprehensive consideration, and its manufacturing precision is also the most difficult to ensure in precision machining, which is the technical bottleneck of the development of the resonator.

\section{Data Availability}

The data required to reproduce these findings cannot be shared at this time as the data also forms part of an ongoing study.

\section{Conflicts of Interest}

The authors declare that they do not have any commercial or associative interest that represents a conflict of interest in connection with the work submitted.

\section{Acknowledgments}

This work was partially supported by the Natural Science Foundation of Shaanxi Province, project no. 2020JM-488, National Program on Key Basic Research Project, project no. 2017YFC0704200, and Special Scientific Research Project of the Education Department of Shaanxi Province, project no. 20JK0728.

\section{References}

[1] F. Delhaye, "HRG by SAFRAN, the game-changing technology," in Proceedings of the 5th IEEE International Symposium on Inertial Sensors and Systems, Moltrasio, Italy, March 2018.

[2] A. Jeanroy, G. Grosset, J. C. Goudon, and F. Delhaye, "HRG by Sagem from laboratory to mass production," in Proceedings of the 2016 IEEE International Symposium on Inertial Sensors and Systems, pp. 1-4, Laguna Beach, CA, USA, February 2016.

[3] P. Yao, T. Qu, K. Yang, and H. Luo, "Hemispherical resonator gyroscop e: current advances and future trends," Navigation Positioning \& Timing, vol. 4, no. 2, pp. 9-13, 2017.

[4] S.-L. Gao and J. Wu, "Drift Mechanisms\&Control of hemispherical resonant gyro," Journal of Projectiles, Rockets, Missiles and Guidance, vol. 28, no. 3, pp. 61-64, 2008.

[5] Z. Fang, S. Liu, and B. Yu, "Sttudy of basic theories of hemispherical resonator gyros," Navigation Positioning\&Timing, vol. 28, no. 3, pp. 61-64, 2008.

[6] V. A. Matveev, M. A. Basarab, and A. V. Alekin, Etal Solide State Wave Gyro, pp. 1-43, National Defense Industry Press, Beijing, China, 2009, in Chinese.

[7] Z. Hong-bo, S. REN, and L. I. wei, "Establishment of dynamics equation of HRG resonator and calculation of natural frequency," Journal of Harbin Institute of Technology, vol. 42, no. 11, pp. 1702-1706, 2010.

[8] C. Yang, C. Chen, W. He, R. Cui, and Z. Li, "Robot learning system based on adaptive neural control and dynamic movement primitives," IEEE Transactions on Neural Networks and Learning Systems, vol. 30, no. 3, pp. 777-787, 2019.

[9] X. Wang, Z. Fang, W. Wu, B. Lou, and L. I. Yun, "Procession analysis of HRG resonator based on two dimensional mass vibrations," Journal of Chinese Inertial Technology, vol. 19, no. 5, pp. 621-626, 2011.

[10] S. Fan, G. Liu, and Z. Wang, "Theory and experimenton hemispherieal resonator gyro," Measurement\&Control Technology, vol. 1, pp. 29-33+2, 1994.

[11] B. Qiu, J. Wang, and P. Li, "Full digital control of hemispherical resonator gyro under force-to-rebalance mode," IEEE Sensors Journal, vol. 15, no. 1, pp. 71-75, 2015.

[12] Z. Xu, G. Yi, Z. Wei, and W. Zhao, "A dynamic modeling method for resonator of hemispherical resonator gyro," Acta Aeron autica et Astronautica Sinica, vol. 39, no. 3, pp. 139-149, 2018.

[13] S. Fan and G. Liu, "Finite element analysis of coupling vibration of Re s nator for HRG," Chinese Journal of Scientific Instrument, vol. 3, pp. 281-287, 1995.

[14] B. Yu, Z. Fang, and C. Jiang, "Finite element analysis based on optimized HRG resonator," Piezoelectrics \&Acoustooptics, vol. 37, no. 4, pp. 561-564, 2015.

[15] B.-C. Shen, Y. I. Guo-xing, S.-Q. Ren, W. Chang-Hong, and Z. Fang, "Finite element analysis on resonator's characteristics of HRG," Journal of Chinese Inertial Technology, vol. 6, pp. 58-62, 2004. 